\title{
LOS PRINCIPIOS DEL SISTEMA LIBERAL DE MEDIOS ANTE LA CRISIS DE LA COMUNICACIÓN PÚBLICA. UNA APROXIMACIÓN CRÍTICA.
}

Xosé Ramón Rodríguez-Polo ${ }^{1}$ : Universidad Rey Juan Carlos en Fuenlabrada. España joseramon.rodriguez.polo@urjc.es

Manuel Martín-Algarra: Universidad de Navarra en Pamplona. España mmalgarra@unav.es

\section{RESUMEN}

Ante el panorama actual de abandono de la función política de los medios y la situación crítica que se cierne sobre el periodismo, consideramos necesaria una reflexión acerca de los primigenios principios normativos sobre los que los medios se desarrollaron vinculándose al progreso de la democracia. En este trabajo realizamos una aproximación a los principios clásicos que alentaron el modelo liberal de los medios. Atendiendo al cambio de mentalidad, a las ideas que sobre la libertad de expresión y la prensa desarrollaron sus principales inspiradores, para esbozar los principios normativos del modelo. Posteriormente desarrollamos una crítica en la que consideramos que el individualismo ha sido uno de los principales causantes de esta crisis, lo que plantea a los medios la urgencia de que el público recupere el sentido comunitario.

PALABRAS CLAVE: Periodismo - Teoría liberal - Sistema de medios - Criticismo de los medios - Democracia

\footnotetext{
${ }^{1}$ Autor correspondiente:

Xosé Ramón Rodríguez-Polo: Profesor de la Facultad de Ciencias de la Comunicación en la Universidad Rey Juan Carlos en Fuenlabrada, España

Correo: joseramon.rodriguez.polo@urjc.es
} 


\title{
THE PRINCIPLES OF THE LIBERTARIAN MEDIA SYSTEM FACED WITH THE PUBLIC COMMUNICATION CRISIS. A CRITICAL APPROACH
}

\begin{abstract}
In view of the actual situation looms over journalism and over the political role of the media, we consider that is necessary make a reflection about the main normative principles in which mass media developed, linking to the democracy progress. In this paper we propose make an approach to the classical principles that encouraged the liberal theory of the mass media. We attend to the culture shift, the ideas in which the freedom of speech and press developed his main inspirators, and we outline the normative principles of the liberal media system. Then we carry out a critical valuation in which we believe that individualism has been one of the main causes of this crisis. To the media, this raises the urgent need for the public that recovers the sense of community.
\end{abstract}

KEY WORDS: Journalism - Liberal theory - Media systems - Media criticism Democracy

"La crisis actual de la democracia occidental es una crisis del periodismo". Walter Lippmann, Liberty and the news, 1920.

\section{INTRODUCCIÓN}

Hace ya unos quince años que Jay Blumer y Michael Gurevitch, atendiendo al exagerado aumento de la trivialización de la información política y la creciente profesionalización de su gestión desde el poder, advirtieron de lo que vinieron a denominar como "la crisis de la comunicación pública" (1995). Era una constatación más del progresivo deterioro de la función política de los periodistas y los medios de comunicación. La prensa, que había sido uno de los principales impulsores de la democracia (Neveu, 2002, p. 23), se convertía ahora en una de las causas más evidentes de su debilitamiento (McNair, 2000, p. 8).

Los requerimientos que la sociedad democrática demanda de la comunicación entraban en contradicción con las estrategias empresariales de los medios. La creciente comercialización (Martín Algarra, 1999; Sánchez-Tabernero 2008, p. 7-60) ha traído como consecuencia el declive de la profesión periodística y el abandono de los valores de servicio al público. Los objetivos empresariales exigen un nuevo tipo de noticias (Mateos- Pérez, 2009; Redondo García, 2010) en el que la información se encuentra sometida a la "supraideología" del entretenimiento (Postman, 1991, p. 91). El espectáculo, el mestizaje de géneros y la supremacía de las emociones (Grandío, 2009, 155) construye un tipo de información fácil, las soft news (Patterson, 2000, p. 3 y ss.), 
donde se abandona el análisis crítico y se banaliza la política. Todo esto ha llevado a algún autor a acusar a los medios de difusión de haberse convertido en una importante fuerza antidemocrática (McChesney, 1999, 48 y ss.).

$Y$ es que la estrecha relación que existe entre democracia y comunicación hace que ambas caminen en dependencia recíproca:

Todas las cosas que colocamos bajo el rótulo de la comunicación - la información, los medios de difusión, las telecomunicaciones, la opinión pública...forman, con la política y la democracia, un entramado de interacciones de gran complejidad. Y, en una perspectiva sistémica, eso significa que el empeoramiento de cualquier elemento perjudica al conjunto, mientras que la mejora de cualquier elemento le beneficia (López-Escobar, 2001, p. 28).

Sin duda son muchos los factores que han influido en la situación actual del periodismo, y las posibilidades tecnológicas actuales sólo han venido a ponerlo todo más en evidencia. No se puede negar que nos encontramos "insertos en la confusión" (Diezhandino 2008, 201) pero, precisamente por ello, consideramos que el periodismo si quiere seguir siéndolo (Tomás Frutos, 2010, p. 3-4) “debe recuperar el enjuiciamiento intelectual de su propia función y de los requisitos teórico-normativos de su actividad" (Dader 2009, 166).

Por ello con este trabajo proponemos volver la vista hacia atrás y reencontrarnos con los principios mismos que alentaron el desarrollo del periodismo y de su función política. Quizás ahí, en el sistema liberal de los medios, podamos encontrar alguna clave de lo que ha fallado que nos encamine en la búsqueda de soluciones.

Parece necesario aclarar que no se puede hablar de "un" sistema liberal. El liberalismo aplicado a los sistemas de comunicación no se ha dado de una sola forma ni en un periodo histórico determinado, como ocurrió con los sistemas autoritarios y totalitarios del siglo XX e incluso con el sistema de las monarquías absolutas (Siebert, Peterson y Schramm, 1956). La aplicación del pensamiento liberal a la difusión de mensajes ha sido distinta según los países y las tradiciones políticas, y ha evolucionado de mayor a menor control por parte de los estados - o viceversadependiendo de variables muy diversas como las circunstancias políticas dentro de cada país, el contexto de las relaciones internacionales, o el desarrollo de las tecnologías de la comunicación. En cualquier caso, parece obvio que los sistemas liberales de comunicación han ido imponiéndose como marco de funcionamiento político en una buena parte del mundo (Hallin y Mancini, 2008, 229 y ss.). Por ello, en este trabajo vamos a centrarnos fundamentalmente en las ideas que explican por qué los sistemas liberales se configuran como lo hacen, y no tanto en el modo concreto que esos sistemas adoptan. 


\section{METODOLOGÍA}

La base metodológica se basa en el análisis de las anteriores escuelas de pensamiento que han tratado el tema que nos ocupa. Se trata de analizar los principales medios existentes hoy y aplicarles un método analítico textual que permita producir inferencias fenomenológicas o estructuras de pensamiento novedoso basado en este tipo de reflexiones que crean un puente entre las ideas anteriores (y sus realidades descritas) y las más modernas.

\section{ANÁLISIS Y DISCUSIÓN}

El origen del liberalismo se explica, como es bien sabido, en el desarrollo de la ciencia que tiene lugar en el Renacimiento ${ }^{2}$. Durante esta época las ciencias naturales, especialmente la Astronomía, alcanzaron un grado de exactitud que influyó de manera muy profunda en los planteamientos filosóficos sobre el conocer humano. La exactitud matemática con que se podía calcular el movimiento de los astros y con que se podían predecir los fenómenos cósmicos hizo que se fuera imponiendo la idea de que el conocimiento humano es capaz por sí mismo de conocer el mundo sin necesidad de acudir a explicaciones que no fueran exclusivamente racionales. El universo funciona de acuerdo con unas leyes reducibles a fórmulas matemáticas exactas. Ya no era necesario acudir a la Biblia o a la fe sobrenatural para conocer el mundo o para explicar su funcionamiento. La razón humana estaba en condiciones de alcanzar por sí misma ese conocimiento y de explicar sus razones.

La idea de la autosuficiencia de la razón humana no se redujo a la explicación de los fenómenos naturales, sino que, por el prestigio alcanzado por la lógica de las ciencias, se convirtió en el modo auténtico de conocer racionalmente. La explicación matemática del comportamiento de los cuerpos celestes y la lógica derivada de ella -la lógica formal- pasaron a ser el único modelo legítimo de explicación racional. Un paso decisivo en la definitiva reducción de lo racional a lo científicamente explicable lo da Descartes. Para él la filosofía deber ser una ciencia real, por lo que debe regirse por los principios del conocimiento científico, por los principios que rigen la lógica matemática que hizo exacta la Astronomía. Para ello es necesario dar con el método que permita encontrar un punto de partida evidente por sí mismo desde el que levantar el edificio del saber filosófico realmente científico. Eso es lo que Descartes propone con la duda metódica de la que concluye la verdad clara y distinta, evidente por sí misma que resume el famoso cogito, ergo sum.

En última instancia, esta reducción de lo racional a lo científico introduce un cambio cultural de primera magnitud: aunque no deja de aceptarse la idea de Dios como Creador, se rompe el vínculo posterior entre lo creado y el Creador. La criatura, una vez comenzada su existencia, no sigue dependiendo de Dios, sino que se rige por unas

\footnotetext{
${ }^{2}$ Para la contextualización y aspectos generales siguen siendo de inestimable ayuda los trabajos ya clásicos de Touchard, Jean (2006). Historia de las ideas políticas. Madrid: Tecnos; y Sabine, George H. (2000). Historia de la teoría política. Madrid: Fondo de Cultura Económica.
} 
reglas que el hombre puede llegar a conocer con la sola razón. Es la reducción de Dios a un mero clock maker, un relojero que fabrica una pieza y la pone en marcha, sin que su concurso sea necesario para el posterior funcionamiento de la máquina. Y el ser humano, como criatura, goza de esa autonomía y capacidad de autorrealizarse, además de poder comprenderse y comprender por una vía racional - esto es, científica, autónoma de la Revelación - todo lo que le rodea. Nos encontramos ante un nuevo concepto de naturaleza humana, radicalmente distinto al heredado de la filosofía cristiana de la Edad Media.

Consecuencia inevitable de esta autonomía de la razón humana es el protagonismo del hombre frente al marco social: cada individuo es un ser racional y libre, capaz de conocer el mundo y valorarlo como bueno o malo con su propia razón. La historia del arte nos ofrece una buena manifestación de este cambio de mentalidad: con el Renacimiento, por ejemplo, se impone el arte de autor y se extingue el arte de escuela.

Por lo tanto, podemos señalar como características básicas del pensamiento liberal el individualismo, esto es, la reducción del hombre a un individuo autosuficiente, y el racionalismo, que reduce la racionalidad a las leyes de la lógica formal. Sobre estos dos presupuestos se construye la doctrina política liberal. La sociedad y el Estado son producto de un pacto que tiene como fin alcanzar el bienestar y la felicidad individuales. Estas se alcanzan por medio del progreso que se logra a su vez a través de la acumulación de opiniones, comportamientos, reflexiones..., individuales. En la doctrina liberal la libertad es un elemento básico. Consecuencia de la consideración del hombre como individuo maduro, con capacidad y derecho a pensar y decidir por sí mismo, la libertad individual es encauzada en la vida social de acuerdo con el principio de laissez, faire, laissez passer (dejar hacer, dejar pasar). Una "mano oculta" hará que esa acumulación de reflexiones, opiniones, ideas y comportamientos produzca el progreso político y económico y que se alcance la verdad.

El ámbito en el que por vez primera se aplican las ideas liberales es en el comercio, en lo que se denominó librecambismo, esto es, la libre circulación de mercancías sin trabas fiscales ni aduaneras. Posteriormente, esas ideas irán fraguando en concepciones de la vida social y política, y también en principios acerca de la racionalidad de la vida social y de la opinión pública (Peters, 2004). El desarrollo de las clases medias, el Bill of rights (1689) en Inglaterra, la Independencia de los Estados Unidos (1776), la Revolución francesa (1789) y el resto de las revoluciones liberales durante el siglo XIX, determinan la definitiva aplicación práctica de las ideas liberales en la política y la economía.

\subsection{La doctrina clásica sobre la libertad de expresión y la prensa}

La aplicación de las ideas liberales a la política lleva consigo la concepción liberal de la prensa y - ya en el siglo XX, conforme vayan apareciendo - los otros medios de comunicación. Por lo que se refiere específicamente a la descripción de la doctrina liberal sobre la prensa, hay que referirse al pensamiento de tres autores: John Milton, John Stuart Mill y Thomas Jefferson. 
John Milton (1606-1674) publicó en 1644 un alegato contra la censura titulado Areopagítica. Por la libertad de imprenta sin censura. En él, Milton aplica el principio librecambista de libre mercado a las ideas y reivindica el principio de autogobierno. Más que una exposición sobre la libertad de prensa e imprenta, en la Areopagítica, Milton hace un alegato contra la censura puritana en la Inglaterra del siglo XVII (Martínez Guerra, 2002, p. 124). Afirma que los hombres, por medio de su razón, son capaces de distinguir lo correcto de lo incorrecto, lo bueno de lo malo. Por eso, para desarrollar su talento, el hombre tiene que poder acceder libremente a las ideas y al pensamiento de los demás. Ese choque con las ideas ajenas le permitirá encontrar la verdad. Más aún, sólo es posible acceder a la verdad de ese modo.

Milton desarrolla dos conceptos básicos en la doctrina liberal sobre la comunicación: el concepto de libre mercado de ideas y el proceso de autogobierno. El libre mercado de las ideas es la aplicación al ámbito gnoseológico de las ideas librecambistas: sólo en la medida en que se permita e incluso se facilite que se viertan a la palestra pública un gran número de ideas y opiniones individuales, se producirá el progreso en el conocimiento, esto es, se alcanzará la verdad. El concepto liberal de opinión pública, tan relevante desde el punto de vista político, está formulado de algún modo en este principio de Milton (Monzón, 1996, p. 54).

El proceso de autogobierno hace referencia a que esa pluralidad de ideas y opiniones no produce desequilibrio alguno, antes bien, la "mano oculta" que, según el pensamiento liberal, rige el funcionamiento de las cosas hará que, como en la economía, el conocimiento se distribuya equilibradamente y se alcancen posiciones racionales, verdaderas.

Desde el punto de vista ideológico, la importancia de John Milton es evidente, ya que formula algunos de los principios básicos de los sistemas liberales de comunicación. Sin embargo, la consistencia de esas ideas en la exposición que hace en su Areopagítica no es grande. Esta obra es más el fruto de una reacción ante la censura de sus escritos que el resultado de una reflexión teórica ante el problema de la censura en general y de la libertad de expresión. Prueba de ello es que Milton no entiende que el principio de la libertad de imprenta sea universal, aplicable a todos. Así, justifica en su obra que la censura sea impuesta, por ejemplo, a los católicos, a los que no considera suficientemente honestos como para permitirles la libertad de prensa sin censura.

John Stuart Mill (1806-1873) en su obra de 1859, Sobre la libertad, formula sus ideas sobre la libertad de expresión basándose en que la libertad es el derecho del individuo maduro a pensar y actuar como quiera mientras no perjudique a los demás. Según Mill, sólo el libre actuar y el libre pensar permiten al hombre alcanzar la felicidad.

Stuart Mill justifica la necesidad de la libertad de expresión en cuatro ideas básicas:

a) Silenciar una opinión puede significar silenciar la verdad.

b) Una opinión equivocada puede contener una parte de verdad que resulte ser 
necesaria para la consecución de la verdad completa.

c) Incluso aunque la opinión aceptada por todos fuera la verdad, habría que garantizar la libertad de expresión, ya que la tendencia general en esos casos es a aceptar esa opinión de un modo no racional. Sólo si existe la necesidad de defender las propias opiniones, éstas se racionalizan y se defienden en razón de su verdad y no por costumbre.

d) La opinión comúnmente aceptada que no es discutida de vez en cuando pierde su vitalidad y su efecto sobre la conducta y el carácter. Es necesario que sea negada o rechazada para que siempre sea una aceptación viva y racional.

Mill da gran importancia a que las opiniones sean aceptadas racionalmente y no como producto de la presión social, la costumbre o la comodidad individual. Para ello es necesario que la opinión pública sea verdaderamente un continuo contraste de opiniones diversas como fruto del cual saldrán posturas racionales y conocimiento verdadero, esto es, lógico, científico (Rey-Morató, 1989, p. 39-47).

Sin embargo, resulta difícil llegar a saber en qué consiste la verdad según Mill. Parece que habla de la verdad en lo político, pero precisamente en este ámbito es claro que pueden aceptarse muchas opciones como verdaderas, aun siendo distintas entre sí. Al mismo tiempo, la afirmación de que el límite de la libertad está en que no se perjudique a los demás, implica que lo que perjudica o no a los demás es algo absoluto, que no surge de la confrontación de ideas, ya que, de lo contrario, el límite de la libertad sería el poder, lo que supondría que la libertad de unos sería la no libertad de otros.

Thomas Jefferson (1743-1826), fiel seguidor de las ideas liberales de Montesquieu y Locke, aporta a la reflexión sobre la libertad de prensa su experiencia como hombre de estado. Su pensamiento se centra en el papel de la prensa en la vida política.

Como Milton y Mill, Thomas Jefferson considera que, aunque un individuo pueda tomar decisiones equivocadas, la sociedad como grupo, representada por la mayoría, no se equivocará. Para que este proceso se cumpla es necesario que la sociedad esté bien educada y bien informada. Y la educación y la prensa podrán cumplir sus papeles en el sistema democrático sólo si están libres del control del Estado.

La prensa, además de la función informativa debe vigilar la actividad del gobierno (watch dog) (Martínez-Albertos, 1994) y denunciar las desviaciones que se produzcan con respecto a las funciones que constitucionalmente tienen los gobernantes. El pensamiento de Thomas Jefferson sobre el papel de la prensa está bien reflejado en su conocida frase en la que afirma que más vale una prensa sin gobierno que un gobierno sin prensa:

The basis of our governments being the opinion of the people, the very first object should be to keep that right; and were it left to me to decide whether we should have a government without newspapers or newspapers without a government, I should not hesitate a moment to prefer the latter (Jefferson, 1787). 


\subsection{Principios del modelo liberal clásico}

En resumen, podemos afirmar que la doctrina liberal con respecto a la comunicación se basa también en la ausencia de restricciones, e incluso de leyes, que organicen o encaucen de algún modo la libertad de expresión de los individuos a través de los medios de comunicación. La prensa - los medios en general - tiene una función política de primera importancia, ya que garantiza la libre circulación de ideas y permite a los ciudadanos la posibilidad de tomar sus decisiones racionalmente.

Refiriéndonos ya a la aplicación práctica de estas ideas a los sistemas liberales de comunicación podemos hablar de cuatro principios definitorios:

a) La libre circulación de la información, sin ningún tipo de restricción por el Estado o las empresas.

b) El libre acceso al acontecimiento, lo cual implica igualdad para conocer lo que ocurre y tiene especial importancia si pensamos en la actividad de los informadores para los que, según este principio, no hay materias reservadas.

c) La libertad de retransmisión, de manera que cualquiera y por cualquier medio pueda difundir información.

d) La libertad de recepción, sin controles ni límites por parte del Estado, de las organizaciones informativas o de cualquiera otra entidad.

Vistas estas características, parece claro que no es posible el desarrollo armónico de un sistema liberal puro, ya que garantizar en toda su extensión cada uno de los principios señalados más arriba implica necesariamente limitar alguno o algunos de los otros. Por otra parte, diversidad de factores impiden esa absoluta libertad de comunicación propia de los sistemas liberales. Factores técnicos como la limitación del éter o de cualquier otro soporte físico para la transmisión de señales. De carácter social como es que no todos los ciudadanos tienen la capacidad o la necesidad de expresarse a través los medios (Peters, 1989). Factores económicos, ya que con frecuencia las leyes del mercado llevan a una dinámica concentradora que hace difícil la supervivencia de las pequeñas empresas frente a las grandes (Iosifidis, 2010). Como ocurre con la historia general, la historia de la comunicación se encarga de demostrar que el liberalismo aplicado sin matices a la organización de la comunicación genera contradicciones que acaban por mermar las libertades individuales y, por tanto, las libertades sociales.

Dadas las limitaciones que acabamos de señalar, las ideas liberales se han aplicado a los medios de comunicación más como una tendencia que como una realidad absoluta. La libertad de comunicación a través de los medios está estrechamente vinculada a la libertad de empresa mercantil, ya que en el sistema liberal la empresa es el soporte más adecuado para la organización de la comunicación colectiva. En este sentido, la libertad de prensa ha seguido los pasos - también con sus limitaciones - de la libertad de empresa. Por otra parte, dentro de los sistemas políticos liberales, se fueron estableciendo limitaciones a la libertad de prensa conforme la experiencia hacía ver a los gobernantes la conveniencia de legislar sobre ese derecho ciudadano con el fin de evitar la comisión impune de delitos. Por ello, en las democracias liberales, sólo cabe la 
limitación y el control de la comunicación por ley y, la aplicación de esas leyes tiene un carácter represivo (una vez cometido el delito), no preventivo.

\subsection{Individualismo, racionalismo, vida social y tarea de los medios}

La historia se ha encargado de demostrar que estos principios del liberalismo individualismo y racionalismo-, producen desequilibrios y contradicciones en el desarrollo de la vida social (Rodríguez-Polo \& Martín-Algarra, 2008, p. 155-157). Ya señaló Boecio en su clásica definición el carácter individual de la persona. Pero el hombre es persona, no sólo individuo. El carácter personal del ser humano lleva inscrita una naturaleza social que hace poco eficaz el esfuerzo por encontrar una felicidad exclusivamente individual: no es posible. La naturaleza social del ser humano hace necesaria la existencia de la "felicidad social" para que la "felicidad individual" sea posible. Existe un vínculo natural entre los hombres. La sociedad no es sólo un pacto entre individuos sino una inevitable manifestación de la naturaleza humana de la que la vida social y la comunicación son pruebas evidentes.

Respecto al otro punto de partida del pensamiento liberal -el racionalismo-, existe, como en el caso del individualismo, una desproporción en el planteamiento. Si bien es cierto que la razón humana es capaz de explicar racionalmente la realidad que nos rodea, no lo es menos que nunca podrá el hombre llegar a conocer en plenitud las cosas, y, por tanto, siempre podrá comprenderlas mejor, con mayor profundidad. Precisamente por ello no hay un único modo -el "racional"- de acceder a la comprensión de la realidad, como parece apuntar el liberalismo. El método científico regido por la lógica formal es un medio para conocer las cosas, pero no el único válido. Además, las distintas esferas de la realidad exigen modos diferentes de conocimiento. La metodología de las ciencias naturales es apropiada para comprender los fenómenos físicos, pero no lo es tanto para conocer realidades y fenómenos de naturaleza no física o no sólo física, como es el caso del hombre y su actuar. La filosofía contemporánea ha planteado con toda claridad los problemas del positivismo y la necesidad de metodologías específicas y apropiadas para las ciencias humanas y sociales.

De las ideas expuestas podría afirmarse que, hasta cierto punto, el sistema liberal -el pensamiento liberal- se caracteriza por una ingenua concepción antropológica. El liberalismo da por supuesto que en un sistema en el que todos los hombres buscan su felicidad individual no habrá conflictos entre ellos, sino equilibrio y progreso. Este naturalismo un tanto romántico olvida la naturaleza limitada del hombre, ya entrevista en la Antigüedad clásica como refleja Ovidio en su Metamorfosis (VII 20-21: video meliora; proboque; deteriora sequor) y asumida en la tradición judeo-cristiana. Esto, sin embargo, no quiere decir que sea imposible ese equilibrio, sino que se trata de un equilibrio arduo para el hombre. El hombre precisa del esfuerzo para reconocer y buscar el bien, y por ello es lógico que, en la búsqueda de la felicidad, que es de lo que trata básicamente la teoría liberal, no todos identifiquen y deseen lo que verdaderamente produce el equilibrio entre la felicidad personal y la justicia social, tal y como postula el liberalismo. 
La aplicación del pensamiento liberal a la comunicación pública ha llevado acarreada un constante cuestionamiento de los medios, acusados de ser los artífices del progresivo empobrecimiento de la vida pública.

Walter Lippmann denunció que los medios menospreciaban la información, el conocimiento cabal de lo que sucede, cayendo en la propaganda y en la exaltación de la opinión (1920, p. 8-9). John Dewey, que compartía los análisis de Lippmann, veía al público como un ente difuso, fácilmente manipulable por los medios, atomizado en multitud de grupos guiados por su propio interés y preso de una creciente apatía para los asuntos públicos. Acuñó la expresión “eclipse del público" para avisar del declive de la base comunitaria de la vida democrática (Dewey, 2004, p. 116). La Comisión para la libertad de la prensa, presidida por Robert Hutchins, en su informe final, A free and responsible press, se veía en la obligación de recordar a los medios que las necesidades informativas de la sociedad debían de convertirse en su objetivo prioritario (1947, p. 126), tanto se habían apartado de su razón social.

El énfasis que el pensamiento liberal puso en el componente individual de la persona, obviando su indisoluble componente social, había inundado la actuación de los medios. Y éstos habían fomentado la pérdida del sentido del interés público, procediendo de manera irresponsable ante la sociedad.

\section{CONCLUSIONES}

Quizás sea precisamente aquí, en el individualismo propugnado por el liberalismo, donde se encuentre el punto de partida erróneo del sistema liberal de medios. Que la comunicación pública deje de estar al servicio del mantenimiento y mejora de la vida social, es decir, que no sirva para compartir objetivos sociales, que no sirva para construir sobre lo que une, manifiesta una contradicción de enorme repercusión. Detrás de ella subyace el debate que sobre el deber ser de la sociedad mantuvo John Dewey contra el liberalismo (Morán, 2009, p. 23). Frente a la compresión de la sociedad como una simple asociación de individuos basada en la cercanía física, lo que él denominaba la "gran sociedad", contraponía la "gran comunidad", una comunidad en la que sus miembros comparten intereses comunes, participando de una unión moral (Dewey 2004, p. 37-138).

Estas observaciones fueron ratificadas por Robert Putnam en su trabajo Bowling alone (2000), la democracia se encontraba en quiebra porque se estaba perdiendo el sentido comunitario y el compromiso cívico, y los medios - en concreto la televisión - eran uno de los motivos principales de la situación.

Los medios, ciegos ante las consecuencias de sus acciones, no han sabido ver que dependen totalmente de la viabilidad de la vida pública. Siguiendo a uno de los autores más lúcidos en su crítica al modelo liberal, James Carey, "el periodismo sólo tiene sentido en relación con el público y la vida pública" (1987, p. 14). Como explica Merrit, "una sociedad desinteresada por los asuntos públicos, replegado hacia la vida y asuntos privados, no tiene necesidad ni del periodismo ni de los periodistas" (1995, p. 10). 
Las palabras de Lippmann escritas hace más de noventa años (1920, p. 4) resuenan con especial contundencia cuando señalan al periodismo como el causante de la crisis de la democracia. Por ello, hoy, la comunicación pública debe asumir con urgencia la tarea de recuperar su función constructora de la vida social. Para conseguirlo deberá volver a situar en el centro mismo de su razón de ser la idea de interés público y de bien común, quizás sea ésta la única manera de reconstruir el público y, como dice Carey, “devolverlo a la existencia" (1987, p. 4).

\section{REFERENCIAS}

Blumer, J. \& Gurevitch, M. (1995). The crisis of public communication. Londres: Routledge.

Carey, J. W. (1987). The press and public discourse, en The Center Magazine, n 20, p. 416.

Commission on freedom of the press, The (1947): A free and responsible press. A general report on mass communication: newspaper, radio, motion pictures, magazines, and books.. Chicago: Chicago University Press

Dader, J. L. (2009). Periodismo en la hipermodernidad: consecuencias cívicas de una identidad débil (y algunas vías de reconstrucción), en Textual $\mathcal{E}$ Visual Media, n 2, p. $147-170$.

Dewey, J. (2004). La opinión pública y sus problemas. Madrid: Ediciones Morata.

Diezhandino-Nieto, M. P. (2008). Un nuevo paradigma para tiempos cambiantes, en Textual \& Visual Media, (1), p. 191-204.

Grandío, M. M. (2009). El entretenimiento televisivo. Un estudio de audiencia desde la noción de gusto". Comunicación y Sociedad, vol. XXII, (2), p. 139-158.

Hallin, D. y Mancini, P. (2008). Sistemas mediáticos comparados. Tres modelos de relación entre los medios de comunicación y la política. Hacer. Barcelona.

Iosifidis, P. (2010): Pluralism and Concentration of Media Ownership: Measurement Issues. Javnost-The Public, vol. 17, (3), p. 5-20.

Jefferson, T. (1787). Carta a Edward Carrington, 16 de enero. Disponible en línea en The online library of liberty. Consultado el 15 de Septiembre del 2011, Disponible en:

http://oll.libertyfund.org/?option=com staticxt\&staticfile=show.php\%3Ftitle=802\&chap ter $=86653 \&$ layout $=$ html\&ltemid $=27$.

Lippmann, W. (2007). Liberty and the news. Princeton: Princeton University Press.

López-Escobar, E. (2001). Comunicación, información y democracia, en Galdón, G. 
(coord.) Introducción a la comunicación y a la información (p. 27-45). Barcelona: Ariel.

Maldonado, M. (2010). Comunicación nomy, crisis funcional de los medios de Comunicación, en Estudios de periodística, nº 7, p. 315-326.

Martín-Algarra, M. (1999): Capitalismo y crisis funcional de los medios de comunicación, en Estudios de periodística, n ${ }^{\circ}$ 7, p. 315-326.

Martínez-Albertos, J. L. (1994): La tesis del perro-guardián: revisión de una teoría clásica, en Estudios del mensaje periodístico, n ${ }^{\mathrm{o}}$ 1, p. 13-25.

Martínez-Guerra, A. (2002): "Areopagítica y su influencia en la Primera Enmienda Norteamericana. Un estudio a través de la jurisprudencia" en Historia y Comunicación Social, no 7, pp. 121-145.

Mateos-Pérez, J. (2009). La información como espectáculo en el nacimiento de la televisión privada española (1990-1994), en Estudios sobre el mensaje periodístico, $\mathrm{n}^{\circ} 15$, $\mathrm{p}$. 315-334.

McChesney, R. W. (1999): Rich media, poor democracy: communication politics in dubious times. University of Illinois Press. Urbana.

McNair, B. (2000). Journalism and democracy: an evaluation of the political public sphere. Londres: Routledge.

McQuail, D. (2000). Introducción a la teoría de la comunicación de masas. Barcelona: Paidós.

Merrit, D. B. (1995). Public journalism and public life. Why telling the news is not enough. Lawrence Erlbaum Associates. Hillsdale (NJ).

Mill, J. S. (1879).On liberty and The subjection of women. Henry Holt and Co. Nueva York. Consultado el 4 de Abril del 2011, Disponible en:

http://oll.libertyfund.org/index.php?option=com staticxt\&staticfile=show.php\%3Ftitle=347\&ltemid=2 $\underline{8}$

Milton, J. (1918) [1644]: Areopagítica. Cambridge: Cambridge University Press.

Monzón, C. (1996). Opinión pública, comunicación y política. Madrid: Tecnos.

Morán, J. G. (2009). John Dewey, individualismo y democracia, en Foro interno, nº 9, p. $11-42$.

Nasón, P. O. (2001). Metamorfosis. Madrid: Cátedra.

Neveu, É. (2002). Four generations of political journalism, en Khun, R. \& Neveu, É. 
(eds.). Political journalism: new challenges, new practices (p. 22-44). Londres: Routledge.

Patterson, T. E. (2000). Doing well and doing good, en Kennedy School of Government. Faculty Research Working Paper Series, RWP01-001.

Peters, J. D. (2004). 'The Marketplace of Ideas': History of the Concept, en Calabrese, Andrew \& Sparks, Colin (eds.): Toward a Political Economy of Culture: Capitalism and Communication in the Twenty-First Century (p. 65-82). Lanham-Maryland: Rowman \& Littlefield

Peters, J. D. (1989). Democracy and American Mass Communication Theory: Dewey, Lippmann, Lazarsfeld, en Communication, vol 11, n 3, pp. 199-220.

Postman, N. (1991) [1985]. Divertirse hasta morir. Barcelona: La Tempestad.

Putnam, R. D. (2000). Bowling Alone. The collapse and revival of American community. Nueva York: Simon and Schuster.

Redondo-García, M. (2010). El valor mediático de la violencia, en Vivat Academia, nº 111. Consultado el 12 de Marzo del 2011, Disponible en: www.ucm.es/info/vivataca/numeros/n111/PDFs/MartaRedocop.pdf

Rey-Morató, J. (1989). La comunicación política. Madrid: Eudema.

Rodríguez-Polo, X. R. \& Martín-Algarra, M. (2008). Medios y democracia: la teoría de la responsabilidad social, en Revista de Comunicación, nº 7, p. 154-166.

Sabine, G. H. (2000). Historia de la teoría política. Madrid: Fondo de Cultura Económica.

Sánchez-Tabernero, A. (2008). Los contenidos de los medios de comunicación. Barcelona: Deusto

Siebert, F. S.; Peterson, T. \& Schramm, W. (1956). Four theories of the press. Urbana: University of Illinois Press.

Tomás-Frutos, J. (2010). Apuesta por la deontología en el tratamiento de las informaciones relativas a sucesos, en Vivat Academia, (112). Consultado el 12 de Marzo del 2011, Disponible en:

www.ucm.es/info/vivataca/numeros/n112/PDFs/JTomascop.pdf (Consultado el $12 / 03 / 2011)$

Touchard, J. (2006). Historia de las ideas políticas. Tecnos. Madrid. 


\section{Xosé Ramón Rodríguez-Polo}

Doctor en Comunicación por la Universidad de Vigo, es Profesor Titular interino en la Facultad de Ciencias de la Comunicación de la Universidad Rey Juan Carlos de Madrid (España), y miembro del Grupo de Estudios Avanzados de Comunicación (GEAC). Sus líneas de investigación son Opinión pública, Comunicación política y Teoría de la Comunicación.

\section{Manuel Martín-Algarra}

Catedrático de Teoría de la Comunicación y de la Información, Vicedecano de la Facultad de Comunicación de la Universidad de Navarra y ex presidente de la Sociedad Española de Periodística (SEP). Su investigación se ocupa principalmente de los fundamentos teóricos de la comunicación. También ha trabajado sobre la comunicación como servicio público y las campañas de comunicación pública, especialmente en el ámbito de la comunicación y salud. 